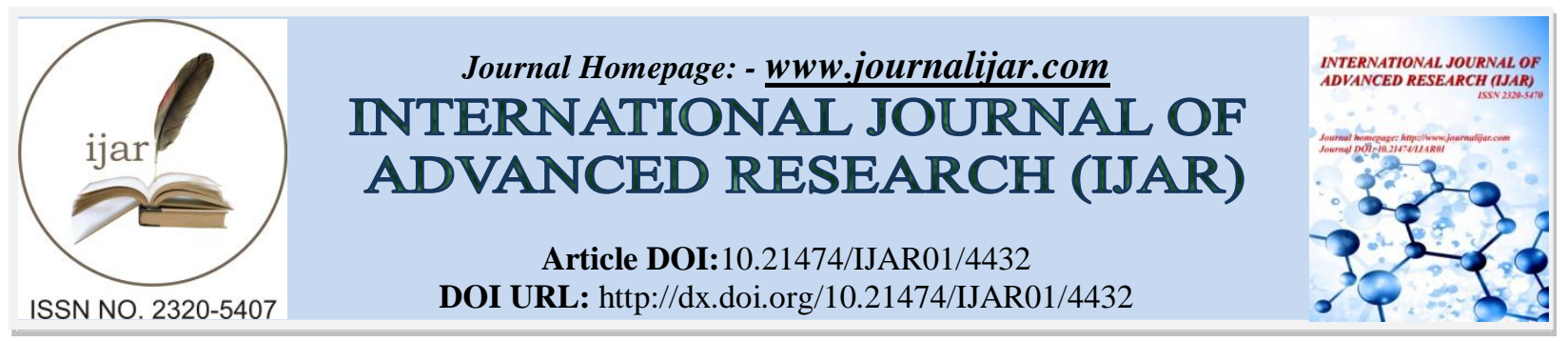

RESEARCH ARTICLE

\title{
COMPARATIVE EVALUATION OF RETENTIVE VALUE OF TOOTH SUPPORTED OVER DENTURE MADE WITH PRECISION AND SEMI-PRECISION ATTACHMENTS.
}

\author{
Nikunj Kumar Patel, Ramesh T R, Soham Mavani, Vilas Patel, Mayurdwaj Mahida and Harjyot Singh \\ Ramgadhiya.
}

\section{Manuscript Info}

\section{Manuscript History}

Received: 10 April 2017

Final Accepted: 12 May 2017

Published: June 2017

Key words:-

Tooth supported overdenture, Semi precision attachment, Precision attachment, Retention

\section{Abstract}

Aim: To evaluate of retentive value of tooth supported over denture made with precision (Pre-fabricated) and semi-precision (Castable) attachments.

Materials and Methods: 24 Acrylic edentulous models were prepared and randomly divided into 2 groups. Group group A consist of Pre- Fabricated Pivots were directly embedded in Acrylic Model with Auto-polymerized Resin. In group B Casting, Finishing \& Polishing of Castable Pivots were done and embedded in Acrylic Model. Denture bases with three loops approximating incisor and molar regions were made for testing in each acrylic models. Yellow retentive caps (Rhein 83) and stainless steel housings (Rhein 83) were embedded in denture base using direct pick up technique. Three chains were attached to the Record base and a 3-point vertical pull was used to determine retention against a vertically directed dislodging force parallel to the path of insertion.

Result: Mean retentive value of Pre-fabricated Attachment/ Precision Attachment group $(4.71 \pm 0.09 \mathrm{~N})$ was higher than mean retentive value of Castable Attachment group $(4.18 \pm 0.12 \mathrm{~N})$. Statistically, significant difference was present in mean retentive values of two groups. $(\mathrm{p} \leq 0.05)$

Conclusion: Within the limitation of this study it can be concluded that use of semi-precision attachment could be cost effective treatment modality without compromising optimal retentive value for tooth supported overdenture

Copy Right, IJAR, 2017,. All rights reserved.

\section{Introduction:-}

Muller De Van stated that Preservation of what remain is more important than the meticulous replacement of what is lost.Preventive Prosthodontics emphasizes the importance of any procedure that can delay or eliminate future Prosthodontic problems and overdenture is an important part as the preventive treatment modality. ${ }^{1}$

Tooth supported overdenture is a removable partial or complete denture that covers and rests on one or more remaining natural teeth. (GPT 8$)^{2}$ Advantages of overdenture are Preservation of alveolar bone, Proprioception, Enhanced stability and retention, Maintenance of vertical dimension of occlusion.

So understanding various facets of tooth supported overdenture and its clinical application should be the goal. 
Various attachments systems are commercially available. In different systems pre-fabricated (precision) \& castable (semi-precision) attachments available. So many studies have been done on retention of precision attachments. But little or no data has been accessible regarding retentive force of castable attachment and Pre fabricated attachment.

So the purpose of the study was to evaluate of retentive value of tooth supported over denture made with precision (Pre-fabricated) and semi-precision (Castable) attachments.

\section{Material and Methods:-}

Preparation of the study Models:-

24 wax models were prepared from class 1 edentulous mould. (Figure 1) Than it was acrylized with heat cure acrylic resin. (Figure 2) Each of the acrylic edentulous model were placed on the surveyor and with help of the drill, two parallel drill placed over the canine region using milling machine. (Figure 3)

\section{Study Design:-}

All models were divide into 2 groups. Each group contains 12 models. Group A (Control Group) having 12 Models with Pre- fabricated Ball and 'O' ring attachments (precision attachments) and Group B (Study Group) having 12 Models with Castable Ball and ' $\mathrm{O}$ ' ring attachments (semi-precision attachments). Both Pre-fabricated \& Castable attachments had similar diameter.

In group A Pre- Fabricated Pivots were directly embedded in Acrylic Model with Auto-polymerized Resin. (Figure 4)

In group B Casting, Finishing \& Polishing of Castable Pivots were done and embedded in Acrylic Model. (Figure 5)

\section{Testing:-}

Over the acrylic model with the help of the cold cure resin denture base were fabricated. Denture bases with three loops approximating incisor and molar regions were made for testing in each acrylic models. (Figure 6)

Yellow retentive caps (Rhein 83) and stainless steel housings (Rhein 83) were used in this study. They were embedded in denture base using direct pick up technique.

Testing carried out in universal testing machine (Instron 3669). Three chains were attached to the Record base and a 3-point vertical pull was used to determine retention against a vertically directed dislodging force parallel to the path of insertion. (Figure 7) The detachment load was measured in newton.

Data were statistically analyzed with $\mathrm{t}$ test.

\section{Result:-}

Table 1:- Relation of retentive value between Pre-fabricated Attachment/ Precision Attachment and Castable Attachment groups

\begin{tabular}{|l|l|l|l|l|l|}
\hline Groups & Number & \multicolumn{2}{|l|}{ Retentive value (Newton) } & t Value & p Value \\
\cline { 3 - 5 } & & Mean & SD & & \\
\hline $\begin{array}{l}\text { Pre-fabricated Attachment//.09 } \\
\text { Precision Attachment }\end{array}$ & 12 & 4.71 & 0.09 & 12.214 & $\leq 0.05$ \\
\hline Castable Attachment & 12 & 4.18 & 0.12 & & \\
\hline
\end{tabular}

Mean retentive value of Pre-fabricated Attachment/ Precision Attachment group (4.71 $\pm 0.09 \mathrm{~N})$ was higher than mean retentive value of Castable Attachment group $(4.18 \pm 0.12 \mathrm{~N})$. Statistically, significant difference was present in mean retentive values of two groups. $(\mathrm{p} \leq 0.05)$ 
Graph 1:-Relation of retentive value between Pre-fabricated Attachment/ Precision Attachment and Castable Attachment groups.

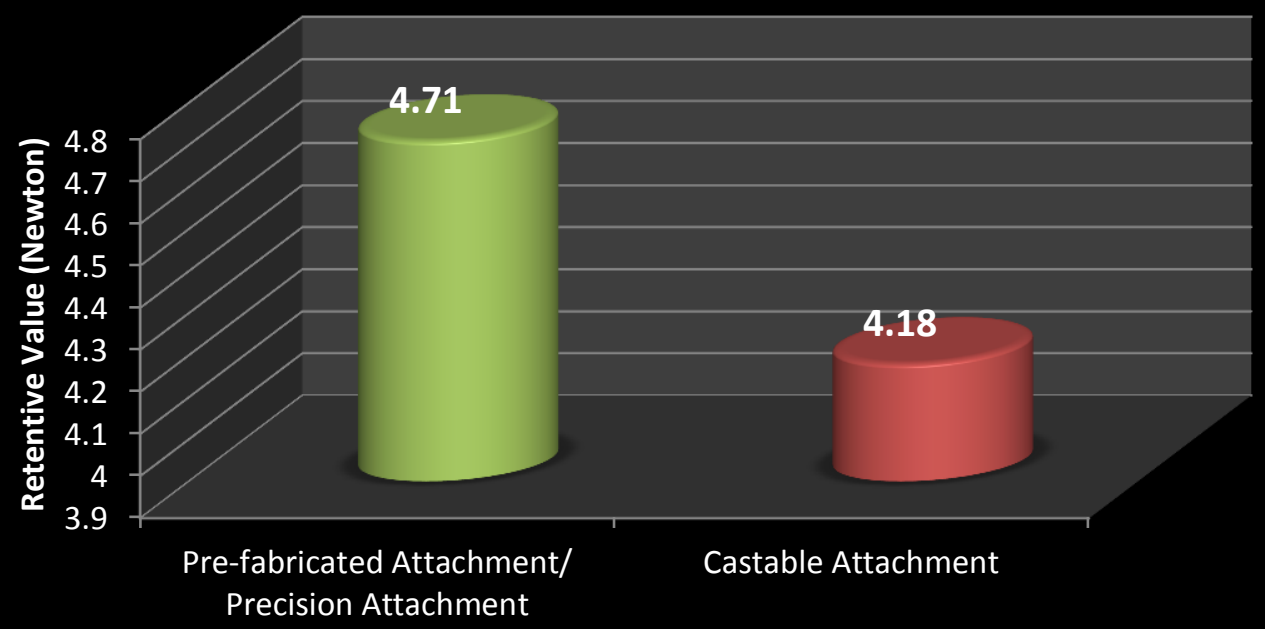

\section{Groups}

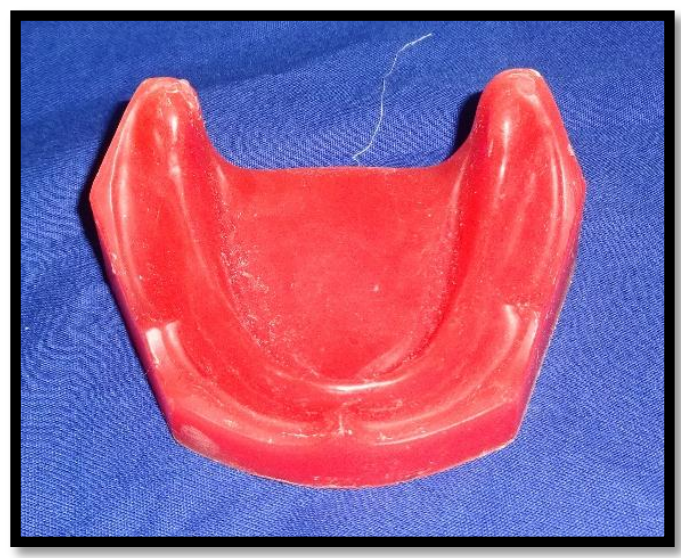

Figure 1:- Wax model prepared from class I edentulous mould.

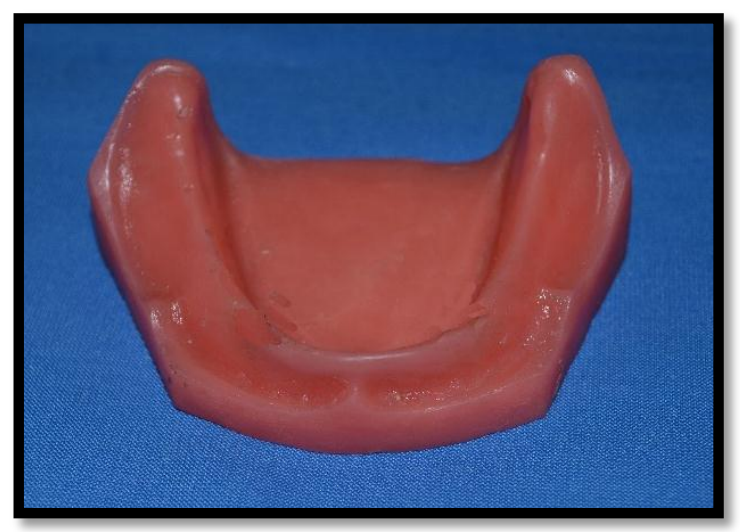

Figure 2:-Acrylization of model with Heat Cure Acrylic resin 


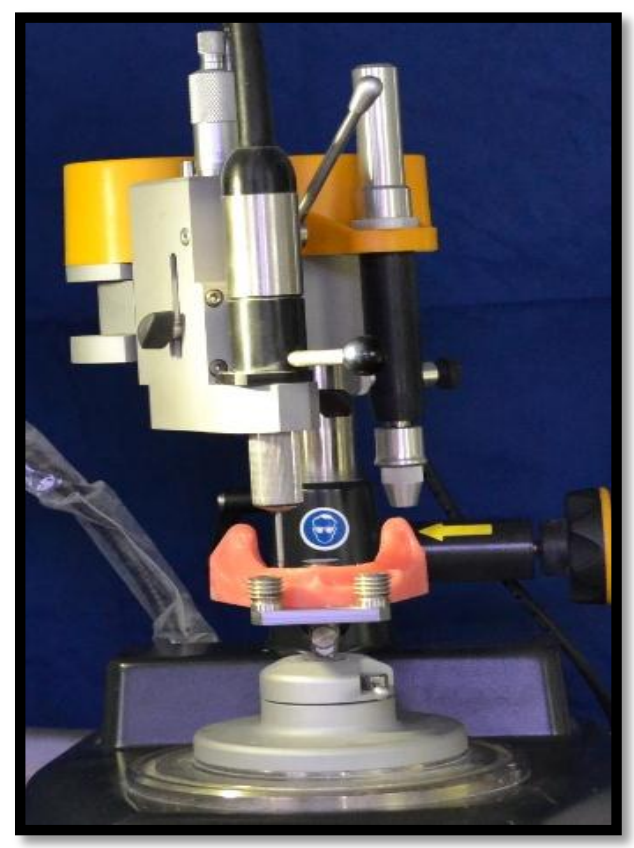

Figure 3:- Two Parallel Vertical Drill place at Canine region using Milling Machine

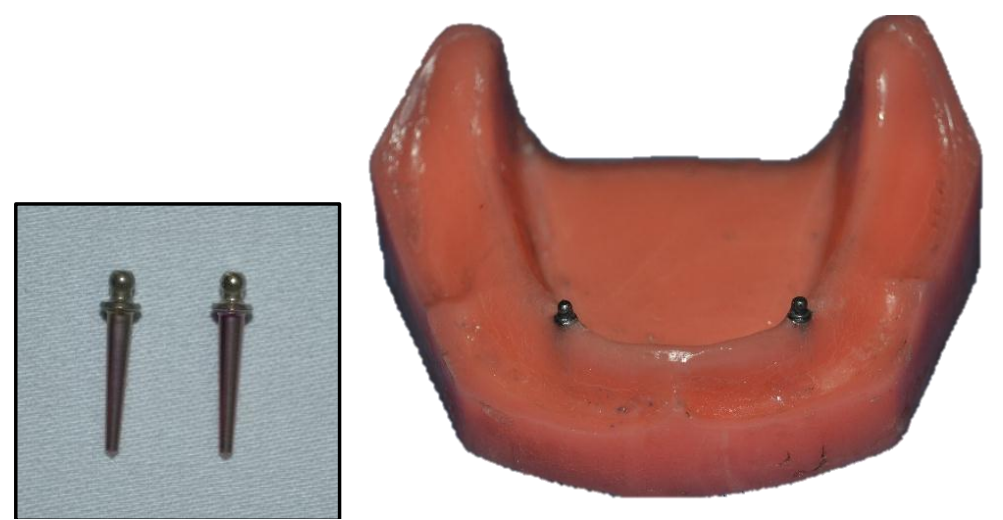

Figure 4:-Pre- Fabricated Pivots were Directly embedded in Acrylic Model with Auto-polymerized Resin (Model A1 to A12).
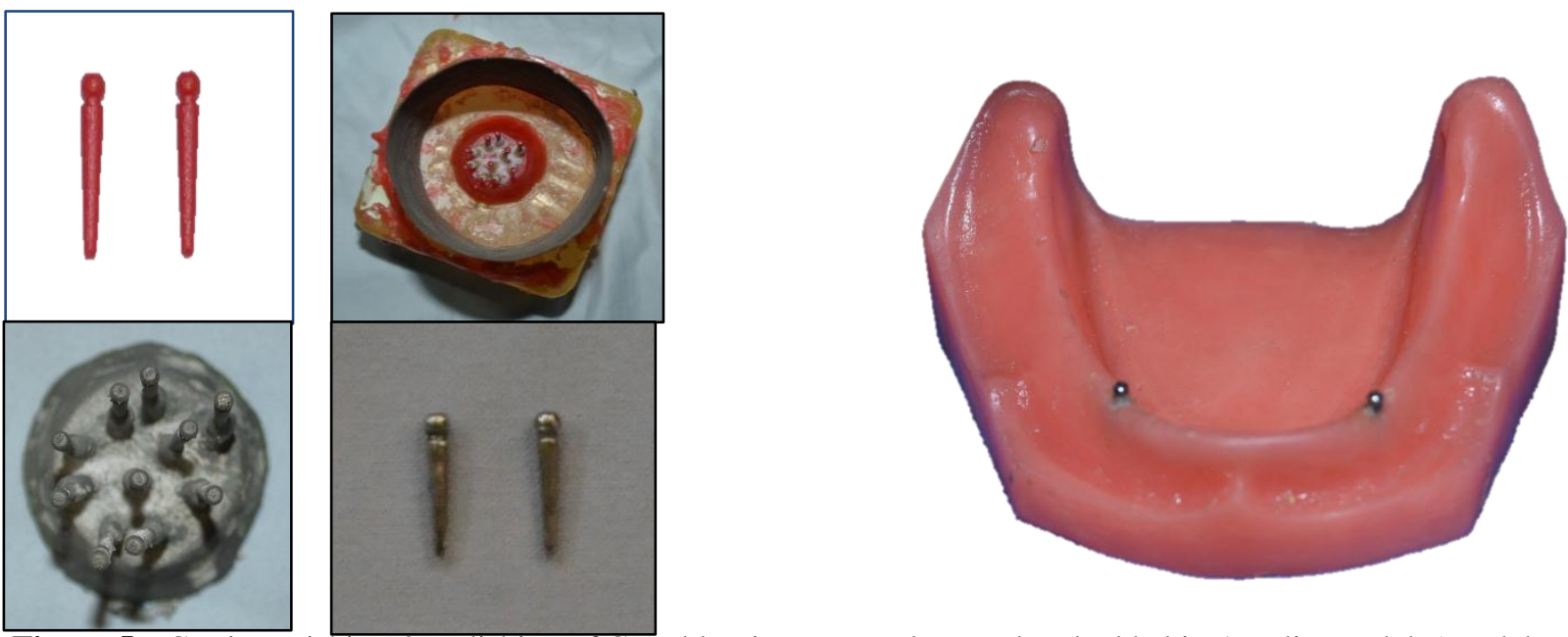

Figure 5:- Casting, Fishing \& Polishing of Castable Pivots were done and embedded in Acrylic Model. (Model B1 to B12). 


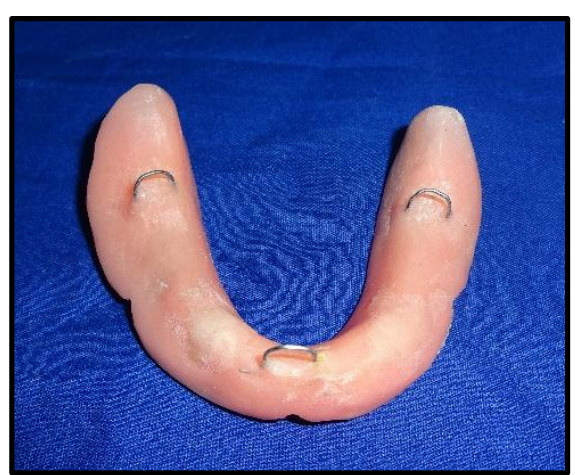

Figure 6:- Denture base with loops.

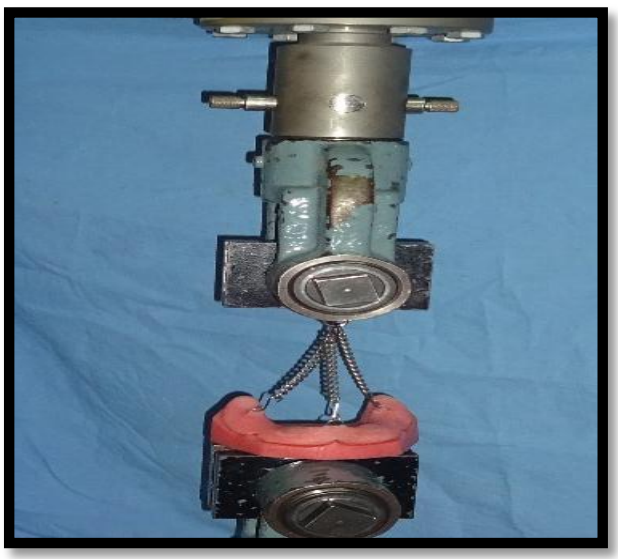

\section{Discussion:-}

Figure 7:-Retention test using universal testing machine.

Attachment has been commonly used to the superstructure of natural teeth \& implant supported overdenture. Use of precision attachments has outstandingly enhanced the retention, esthetics, and patient comfort with less post insertion adjustments. Majority of overdenture are made with pre-fabricated attachment (precision attachment).

Semi-precision attachment such as Castable attachment can be use as alternative to precision attachment mainly for economic reason and custom fit of root canal. ${ }^{3}$ In this study retentive value of Precision and Semi precision was evaluated and compared.

Graph 1 showed that semi- precision attachment provide optimal retentive value that can be compare with precision attachment. Though, retentive value of semi-precision attachment were slightly less than pre-fabricated precision attachment, it was with in satisfactory range. Less retentive value of semi-precision attachment was attributed to limitation of casting accuracy.

Chin-Chuan Fu, Yung-Tsung Hsu demonstrated that prefabricated attachments had superior retention than the cast groups over time. Reduced dimension did not reduce the retention when compared to the cast group. ${ }^{4}$

Kwok- hung chung et al divided the attachments into different category as per the retention. ERA gray into high, Locator LR White, Spheroflex ball, Hader bar and clip, ERA White into medium, Locator Pink into low, Shiner magnet, maxi magnet, magnedisc magnet into very low retention. ${ }^{5}$

Mariko kobayashi compared the retentive force of $\mathrm{O}$ ring and several retained system for one piece root form implant retained overdenture and found that when the medium sized O ring, PMMA and HC were used, an appropriate retention were obtained for the ND implant retained overdenture. ${ }^{6}$

Juergen Setz, Dr Med Dent, Seok Hyung Lee, Dr Med Dent and Eva Engel, Dr Med Dent done a study on retention of prefabricated attachments for implant stabilized overdentures in the edentulous mandible and found that 
Prefabricated attachments for implant-supported overdentures offer a wide range of different forces Under in vitro conditions most of the attachments demonstrated little wear. Some attachments show an increase of retentive forces during the first removals. There are some hints that currently available in vitro wear machines do not properly simulate loss of retention that appears under clinical conditions. ${ }^{7}$

\section{Conclusion:-}

Within the limitation of this study it can be concluded that use of semi-precision attachment could be cost effective treatment modality without compromising optimal retentive value for tooth supported overdenture.

\section{References:-}

1. Winkler S. Essentials of Complete Denture Prosthodontics, 2nd ed. New Delhi: AITBS Publishers; 2006. p. 113.

2. Glossary of prosthodontics terms - 8. J Prosthet Dent 2017;1:10-92.

3. Preiskel HW. Precision attachments in prosthodontics: Overdenture and telescopic prostheses. Vol. 2. London: Quintessence Publishing Co Ltd; 1985.

4. Chin-Chuan Fu, Yung-Tsung. A Comparison of Retention Characteristics in Prefabricated and Custom-Cast Dental Attachments. Journal of Prosthodontics 2009;18: 388-392.

5. Kwok- hung chung, Retention characteristics of attachment systems for implant overdentures. J Prosthodont 2004;13:221-226.

6. Mariko kobayashi. Comparing the retentive force of $\mathrm{O}$ ring and several retained system for one piece root form implant retained overdenture. Prosthodont Res Pract 2006;5:207-213.

7. Juergen Setz, Dr Med Dent, Seok Hyung Lee, Dr Med Dent, and Eva Engel, Dr Med Dent. Retention of prefabricated attachments for implant stabilized overdentures in the edentulous mandible: An in vitro study. J Prosthet Dent 1998;80:323-9. 